\title{
Orbital Cavernous Hemangioma With Proptosis in a Child
}

\author{
Hyokrae Cho
}

\begin{abstract}
Vascular malformations are the most common space-occupying lesions of the orbit in children. Cavernous hemangiomas are the most common benign orbital tumors of adults, while capillary hemangioma is the most common in childhood. A 15-year-old boy was presented with a mass in the right orbit, causing unilateral painless proptosis. Although preoperative computed tomography (CT) scan and magnetic resonance imaging (MRI) are important to diagnose this disease entity, it is difficult to differentiate vascular malformations from other lesion. Right zygomatico-orbital craniotomy was performed to remove the orbital mass. Histological examination showed characteristics of cavernous hemangioma.
\end{abstract}

Keywords: Cavernous hemangioma; Orbital tumor; Proptosis

\section{Introduction}

Cavernous hemangiomas are usually encountered in the orbit as primary tumors in adults. Patients with orbital cavernous hemangiomas typically present in the fourth and fifth decade of life. Lesions are rare in childhood [1].

On the other hand, capillary hemangioma is the most common benign orbital tumor in children. Imaging modalities most often used to evaluate these lesions include computed tomography (CT) and most importantly magnetic resonance imaging (MRI).

This article reports a case of orbital cavernous hemangioma in a 15-year-old boy.

\section{Case Report}

A 15-year-old boy was referred to the neurosurgical depart-

Manuscript accepted for publication March 19, 2015

Department of Neurosurgery, Kosin University Gospel Hospital, 262 Gamcheon-ro, Seo-gu, Busan 602-702, Korea.drchr@hanmail.net

doi: http://dx.doi.org/10.14740/jmc2109w ment for evaluation of a mass in the right orbit. There was no positive family history of hemangioma. Also there was no history of trauma, seizure or focal neurological deficits.

The physical examination revealed no pathologic findings with exception of a painless proptosis in the right eye, causing incomplete closure of the eyelid only after he fell asleep. The ophthalmological evaluation showed that both eyes were equal in size, and eye motility was normal. No strabismus was recognized. Direct ophthalmoscopy showed no abnormality in either anterior segment. The fundus examination in both eyes was normal.

Preoperative MRI of the orbit showed an intraconal intermediate signal mass of pyramid shape in the mediosuperior compartment of right orbit, encasing the optic nerve. The size of lesion was measured $26.5 \times 15.3 \times 23.1 \mathrm{~mm}$ in length. The lesion was separated from the extraocular muscles and showed mild contrast enhancement (Fig. 1).

Right orbito-zygomatic frontal craniotomy was performed to remove the orbital mass. At surgery red-purple, well-defined and pyramid-shaped mass was found and carefully removed with minimal bleeding. The excision was proved to be incomplete, because the lesion encased the optic nerve. The postoperative course was not uneventful.

Histology revealed a vascular malformation composed of some dilated vascular vessels lined by flat endothelium and separated by fibro-connective tissue stroma. The pathological diagnosis was cavernous hemangioma (Fig. 2).

\section{Discussion}

The vast variety of ocular and orbital tumors involves the pediatric orbit and can present with various clinical manifestation. Among vascular lesions, capillary hemangioma is most benign orbital tumor in children [2].

Otherwise, cavernous hemangiomas are the most common benign tumors in adults. They are seen more frequently in female, with peak incidence in early middle age [1]. Patients with orbital cavernous hemangioma usually present in the fifth decade of life [3]. They often result in proptosis, pain, swelling, motility disturbance, visual impairment and disfigurement.

Although orbital tumor may be histologically benign, it can encroach on intraorbital or adjacent orbital structures and 

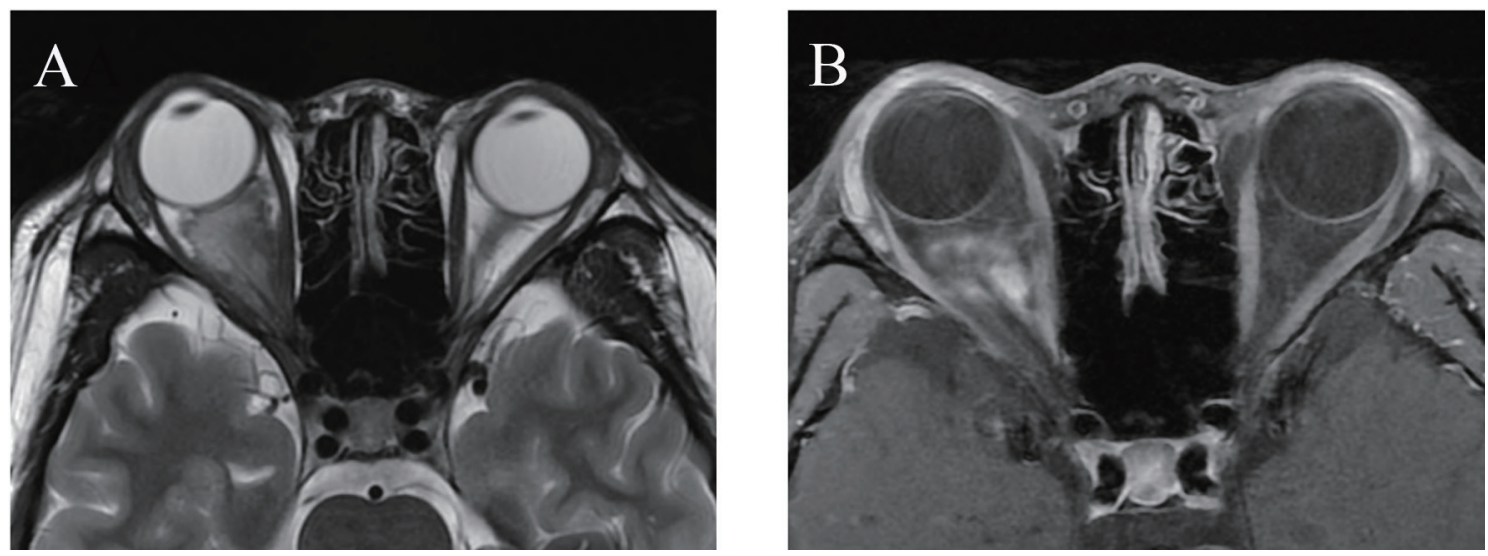

Figure 1. Axial T2-weighted image (A) and postcontrast T1-weighted image (B). A slightly irregular large intraconal mass of pyramidal shape leads to proptosis. The extraocular muscles are displaced and stretched. The irregular enhancement inside the cavernous hemangioma is seen.

be considered anatomically or positionally malignant.

The main differential diagnoses in pediatric orbital tumors include dermoid cyst, vascular tumors, inflammatory disease, mesodermal tumor, neurogenic tumor, metastatic carcinoma and lymphoproliferative disease [2].

Preoperative studies are used to judging pathology of orbital tumors. Imaging is also indicated to assess for the size of the lesion and its relationship with adjacent structures.

On CT images, cavernous hemangiomas are typically well-circumscribed, homogenous and ovoid. The majority of lesions occur at the lateral part of the intraconal space and are located predominantly lateral to the optic nerve in the intraconal space. Conal and extraconal cavernous hemangiomas are rare. Cavernous hemangiomas tend to displace and surround adjacent structure, such as extraconal muscles and the optic nerve, rather than cause direct invasion [4].

On multi-phasic dynamic contrast CT, orbital cavernous hemangioma enhances poorly and heterogeneously in the early arterial and early venous phases because of their low-flow arterial supply. Slow progressive accumulation of contrast contin-

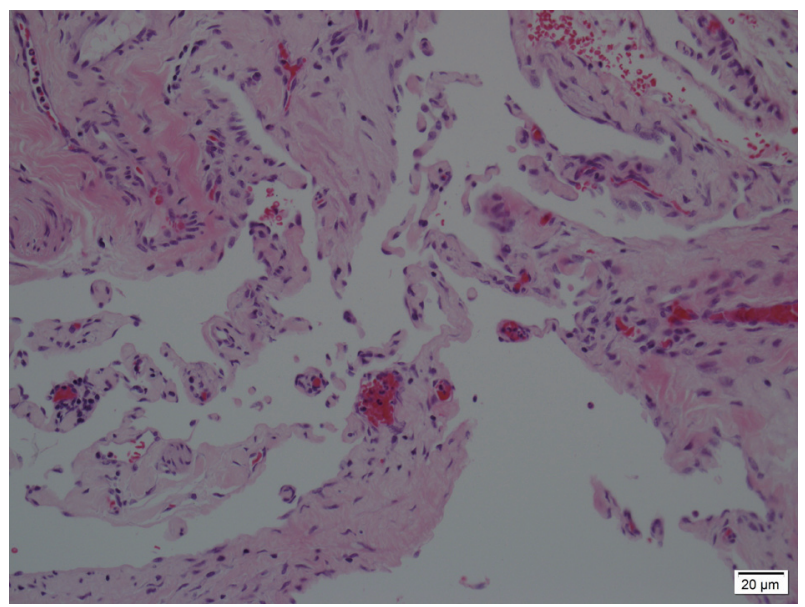

Figure 2. Histological examination revealing a cavernous hemangioma $(H \& E$ staining, original magnification $\times 200$ ). ues within the dilated vascular spaces filling in centrally during the late venous phase [5].

If further characterization is needed, MRI offers more sensitive evaluation of deeper lesions and their relationship to adjacent structures while avoiding the ionizing radiation of CT. Typical hemangiomas are isointense relative to muscle on T1-weighted images, increased signal on T2-weighted images, with no flow voids. Internal septations may be identified on T2-weighted images [4].

Although preoperative CT scan and MRI are important to diagnose this disease entity, it is difficult to differentiate vascular malformations from other pediatric orbital lesions. Furthermore, there is much limitation on applying these studies to an uncooperative child.

At the prospective study of adult cavernous hemangiomas, Scheuerle et al revealed that combination of clinical sign and MRI was highly sensitive and effective for the diagnosis of orbital cavernous hemangioma [3]. In contrast with the most radiological characteristics of cavernous hemangioma, our present case was pyramid shape and encased the optic nerve. Author was hard to consider cavernous hemangioma based on age group, preoperative MRI finding and clinical sign.

The surgical approach used is reliant upon the location and size of the tumor with the surgeon's skill and experience, lending itself to the choice of surgical entry into the orbit. Recently, Boari et al described two surgical approaches according to the location of the cavernous malformation. They used a topographic classification of the lesions within the orbit. Those approaches included a lateral orbitotomy and fronto-orbito-zygomatic approach [6].

As mentioned above, orbital tumor of this case was located in the sector B including orbital apex (Fig. 3). Author chose transcranial fronto-orbital zygomatic approach for removal of orbital tumor.

\section{Conclusion}

Although cavernous hemangioma is a common benign tumor of adults, it should be considered in the differential diagnosis 


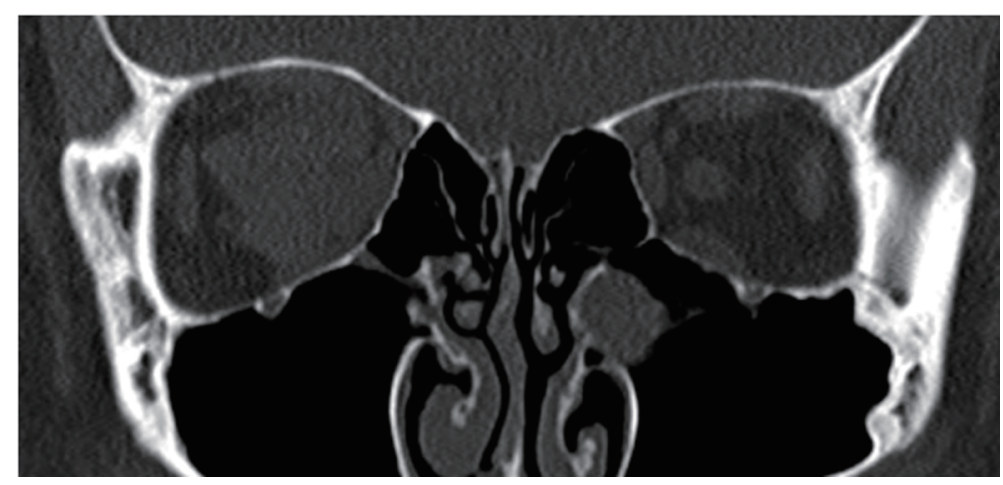

Figure 3. A CT showing a large soft tissue mass in the mediosuperior compartment of right orbit. The cavernous hemangioma encases the right optic nerve.

of a child with unilateral painless proptosis. Intraconal lesions encasing the optic nerve are unamenable to complete excision. Special attention is required in typical imaging appearance and treatment of child cavernous hemangioma.

\section{References}

1. Harris GJ, Jakobiec FA. Cavernous hemangioma of the orbit. J Neurosurg. 1979;51(2):219-228.

2. Castillo BV, Jr., Kaufman L. Pediatric tumors of the eye and orbit. Pediatr Clin North Am. 2003;50(1):149-172.

3. Scheuerle AF, Steiner HH, Kolling G, Kunze S, Aschoff
A. Treatment and long-term outcome of patients with orbital cavernomas. Am J Ophthalmol. 2004;138(2):237244.

4. Tailor TD, Gupta D, Dalley RW, Keene CD, Anzai Y. Orbital neoplasms in adults: clinical, radiologic, and pathologic review. Radiographics. 2013;33(6):1739-1758.

5. Ansari SA, Mafee MF. Orbital cavernous hemangioma: role of imaging. Neuroimaging Clin $\mathrm{N}$ Am. 2005;15(1):137-158.

6. Boari N, Gagliardi F, Castellazzi P, Mortini P. Surgical treatment of orbital cavernomas: clinical and functional outcome in a series of 20 patients. Acta Neurochir (Wien). 2011;153(3):491-498. 